\title{
Intervención oportuna en prematuros extremos: dos casos tratados en Terapia Ocupacional, Toluca, México
}

Timely intervention in extreme prematurity: two cases treated in Occupational Therapy, Toluca, Mexico

Esquivel - González Xochitt ${ }^{1,2}$, Martínez-Cosme Arturo'

Filiación:

${ }^{1}$ Licenciatura en Terapia Ocupacional, Facultad de Medicina de la Universidad Autónoma del Estado de México, México.

${ }^{2}$ Centro Terapéutico del Neurodesarrollo "Bätsi Autismo", México.

Correspondencia: $\square$ MartínezCosme Arturo. Correo electrónico: Ito_amcosme@hotmail.com

Financiamiento:

Ninguno

Conflictos de Interés:

Ninguno

Forma de citar: Esquivel-González $X$, Martínez- Cosme $A$. Intervención Oportuna en Prematuros Extremos: dos casos tratados en Terapia Ocupacional, Toluca, México. Rev Ter. 2021;15(2): 63-70.

Abreviaturas: Terapia Ocupacional, TO; Unidad de Cuidados Intensivos, UCIN.

Fecha de recepción: 23 de febrero del 2021.

Fecha de aceptación: 22 de abril del 2021.

\section{Resumen}

El "prematuro extremo" representa un gran reto en la Unidad de Cuidados Intensivos (UCIN), siendo prioridad la vida en dicho periodo. A su egreso de la UCIN, se enfrenta a un nuevo desafío. Se presentan dos casos de prematuros extremos de 26 semanas de gestación atendidos en el servicio de Terapia Ocupacional. El abordaje de intervención fue Neurodesarrollo y Modelo de Ocupación Humana, los indicadores de evaluación: Evaluación Neurológica para niños menores de cinco años en el primer y segundo nivel de atención, la Evaluación del Desarrollo Infantil (EDI) y Cuestionario Volicional Pediátrico (CVP) y la observación clínica. Promover el desempeño ocupacional en el niño posterior a una estancia prolongada en la UCIN, implica un reto en el abordaje, se debe buscar el proceso de interacción social, intencionalidad a través de la participación en experiencias con significado. En la actualidad, una de las metas para la consolidación de campos de conocimiento, atención y prospectiva es profundizar en la intervención que realiza el respectivo profesional en la UCIN.

Palabras clave: terapia Ocupacional, desarrollo infantil, rendimiento psicomotor, lactante extremamente prematuro.

\section{Abstract}

The "extreme prematurity" represents a great challenge in the Intensive Care Unit (NICU), with life being a priority during this period. Upon leaving the NICU, you face a new challenge. Two cases of extreme premature babies of 26 weeks of gestation treated in the Occupational Therapy service are presented. The intervention approach was Neurodevelopment and Human Occupation Models, the evaluation indicators are: Neurological Evaluation for children under five years of age in the first and second level of care, the Child Development Evaluation Test (CDE) and Pediatric Volitional Questionnaire 
(PVQ) and clinical observation. Promoting occupational performance in the child after a prolonged stay in the NICU implies a challenge in the approach, the process of social interaction must be sought, intentionality through participation in meaningful experiences. At present, one of the goals for the consolidation of fields of knowledge, care and prospects is to deepen the intervention carried out by occupational therapist in the NICU.

Keywords: occupational Therapy, child development, psychomotor performance, extremely premature infant.

\section{Introducción}

Desde su inicio, la terapia ocupacional (TO) ha actuado en ambientes intrahospitalarios. En salud, se han identificado factores de riesgo biológico como el nacimiento prematuro, cuya progresión y desarrollo está condicionado por factores contextuales que permitan el acceso a una intervención oportuna y sistemática ${ }^{1}$.

La Organización Mundial de la Salud (OMS)², reporta una tasa de nacimientos prematuros (5\% y el $18 \%$ ), los clasifica según edad gestacional, siendo los prematuros extremos aquellos con menos de 28 semanas de gestación (SDG); muchos de ellos con algún tipo de discapacidad. La UNICEF, citado por el Instituto Nacional de Pediatría ${ }^{3}$, propone que los recién nacidos (RN) prematuros deben recibir atención con una visión de futuro. El Instituto Mexicano de Seguridad Social ${ }^{4}$, recomienda la intervención oportuna y adecuada, previa detección de los factores de riesgo de morbilidad neurológica con el propósito de corregirlos o limitarlos.

La atención temprana es, el conjunto de intervenciones planificadas, dirigidas a la población infantil de 0 a 6 años, la familia y al entorno, cuyo objetivo es dar atención inmediata a las necesidades transitorias o permanentes que presentan los niños que tienen el riesgo de padecerlos ${ }^{5}$.

En el "prematuro extremo" representa un gran reto en la UCIN, por todas las complicaciones potencialmente a desarrollar, siendo prioridad la vida en dicho periodo, pues las funciones y estructuras corporales presentan inmadurez. El promedio de estancia hospitalaria es de 3 a más de 30 días, y depende de diversos factores (comorbilidad, el grado de severidad y sus complicaciones) $)^{6,7}$.

La TO se centra en el cliente para promover la salud y el bienestar a través de la ocupación8. El proceso de evaluación como punto de partida es necesario, para una adecuada planificación e intervención. A su egreso de la UCIN el prematuro extremo se enfrenta a un nuevo desafío. El terapeuta ocupacional en neurodesarrollo debe tener una visión holística para identificar las interferencias del desarrollo en los periodos críticos, sensibles y ser un facilitador de la adaptación del niño en el entorno ${ }^{9}$. Buscar de manera inicial, conforme al tiempo y evolución del cliente, el desarrollo simultáneo y progresivo con respecto a los hitos del neurodesarrollo. Desde el Marco de Trabajo para la Práctica de TO, se propone la intervención en destrezas de ejecución (sensoriales, perceptuales, motoras, de regulación emocional, comunicación), entre otros ${ }^{7,10}$. A continuación, se presentan dos casos de prematuros extremos atendidos en el servicio.

\section{Reporte de casos}

Antecedentes de ingreso al servicio de Terapia Ocupacional: la tabla 1 describe los antecedentes al ingreso de dos casos de prematuros extremos atendidos en el servicio de TO, así como sus hallazgos clínicos al egreso de la UCIN. 


\section{Valoración Inicial y de seguimiento} en el servicio de Terapia Ocupacional: Los instrumentos de evaluación al inicio y en el seguimiento fueron: Evaluación Neurológica para niños menores de cinco años en el primer y segundo nivel de atención, la Evaluación del Desarrollo Infantil EDI y Cuestionario Volicional Pediátrico (CVP) y la observación clínica (ver tabla 2) ${ }^{11-13}$.

\section{Intervención de Terapia Ocupacional:}

Por lo que, para el primer caso se brindaron dos sesiones semanales y para el segundo caso una sesión semanal ambos con duración de 50 minutos cada una. El Marco de referencia de trabajo fue Neurodesarrollo (control postural estático y dinámico) en las actividades según la edad de desarrollo a estimular y el Modelo de Ocupación Humana, buscando en lo posible el desarrollo volicional (exploración, competencia y logro). El número de sesiones fue determinado por los padres y aspectos socioeconómicos.
Resultados de la intervención: Ambos casos han tenido una evolución significativa y mejora de la participación ocupacional, considerando que los objetivos de la intervención de terapia ocupacional en neurodesarrollo están orientados a promover el aprendizaje y también el desarrollo de habilidades sensoriomotoras, con la intención de facilitar el desempeño de la persona en las áreas de ocupación que conforme a la edad le corresponden.

Los factores de riesgo biológico y la afección en sistemas sensoriales repercuten elocuentemente en el neurodesarrollo, ya que, el sistema visual, auditivo son estructuras cuya función tiene implicancia en el control motor voluntario y la modulación de este. Compensar la edad cronológica y de desarrollo en ambos, sigue siendo un reto, sin embargo, la intencionalidad de los niños ha mejorado. La etapa sensoriomotora en el niño es determinante, por lo que, incrementar la exploración del ambiente y favorecer el sentido de competencia, permite que el niño logre buscar nuevos desafíos. (ver tabla 3 y figuras 1 y 2 ).

Tabla 1. Descripción de los casos atendidos en Terapia Ocupacional

\begin{tabular}{lll|}
\hline \multicolumn{1}{|c|}{ Indicador } & \multicolumn{1}{c|}{ C1 } & \multicolumn{1}{c}{ C2 } \\
\hline Semanas de Gestación & 26 & 26 \\
Peso al nacimiento /talla & $850 \mathrm{~g} / 34 \mathrm{~cm}$ & $850 \mathrm{~g} / 34 \mathrm{~cm}$ \\
APGAR & 7,9 & 2,7 \\
Estancia en UCIN & 122 días & 90 días \\
& & Parto gemelar \\
Factores de riesgo de daño & Síndrome de dificultad respiratoria (SDR), & Diabetes gestacional \\
neurológico & Hiperbilirubinemia & Hematoma retropacentario desde \\
& & el primer trimestre. \\
Atención clínica necesaria & Pediatría, Neurología Pediátrica, Neumología & Pediatría, Neurología Pediátrica, \\
posterior al egreso & Pediátrica, Oftalmología, Audiología & Neumología Pediátrica \\
& Hipoacusia severa & \\
Hallazgos clínicos posterior & Estrabismo convergente & Atrofia prefrontal izquierda \\
al egreso & Desmielinización de predominio derecho en vía visual. & \\
& Reflujo gastroesofágico & \\
\hline
\end{tabular}

Elaboración propia, fuente expediente clínico. 


\section{Tabla 2 Evaluación inicial de los prematuros extremos por parte de TO}

\begin{tabular}{l}
\hline \\
\hline \\
Evaluación \\
Neurológica para \\
niños menores de \\
cinco años en el \\
primer y segundo \\
nivel de atención. \\
(11)
\end{tabular}

\section{Sesión de primera vez.}

\section{Evaluación del Desarrollo Infantil (EDI) ${ }^{(12)}$}

Inicial

$\begin{array}{ll}\begin{array}{l}\text { Cuestionario } \\ \text { Volicional } \\ \text { Pediátrico (CVP) }{ }^{(13)}\end{array} & \text { - Pasivo (1) } \\ & \bullet \text { Dudoso (2) } \\ & \bullet \text { Involucrado (3) } \\ \text { Inicial } & \text { - Espontáneo (4) } \\ & \bullet \text { No aplicable (0) }\end{array}$

${ }^{*}$ Criterios de normalidad

\section{C1}

Criterios de clasificación del Grupo B (desde los dos meses de edad hasta 1 día antes de cumplir 5 meses)

y anormalidad.

Estado de alerta: Anormal

Postura de Reposo: Anormal

Simetría de la expresión facial: Normal

Seguimiento ocular y movimientos oculares: Anormal (Estrabismo convergente, Nistagmus)

Reflejo Cocleopalpebral: Anormal

Tono pasivo: Anormal, hipertonía de predominio en miembros inferiores (Ashworth 3)

Tono activo: Anormal en sostén cefálico.

Reflejos primitivos: Moro, prensión plantar disminuidos.

Edad cronológica: 8 meses de edad.

Edad corregida: 6 meses 1 semana

Grupo 5 que corresponde por edad corregida:

Factores de riesgo biológico: Cinco (Enfermedades sistémicas de la madre, Gestación menor a 34 SDG, peso menor a $1,5 \mathrm{~kg}$, retardo en la respiración, hospitalización en UCIN)

Exploración neurológica: Alteración en la movilidad de su cuerpo, asimetría en AMARILLO: Rezago en el Desarrollo

VERDE: Desarrollo Normal

\section{la movilidad ocular.}

Señales de alerta: Hipertonía, Reflejos primitivos disminuido (Moro), succión alterada, mecánica de la deglución alterada, desarrollo general inadecuado.

Áreas del desarrollo: Motor grueso, motor fino, lenguaje, social; ROJO para todos.

Edad de desarrollo por desempeño: 0 1 mes

Puntuación máxima: 2 Dudoso (Intenta cosas nuevas, práctica habilidades)

Puntuación media: 1 Pasivo (Muestra curiosidad; inicia acciones, se orienta hacia un objetivo; muestra preferencias; permanece involucrado; trata de producir efectos).

Ítems no aplicables: Expresa sus logros, resuelve problemas, busca desafíos, modifica su ambiente, usa la imaginación

Espacio de observación: Natural, interior, tranquilo.
C2

Estado de alerta: Anormal

Postura de Reposo: Anormal

Simetría de la expresión facial: Normal

Seguimiento ocular y movimientos oculares: Anormal (Estrabismo convergente,)

\section{Reflejo Cocleopalpebral: Anormal}

Tono pasivo: Anormal, hipotonía de las cuatro extremidades

Tono activo: Anormal en sostén cefálico.

Reflejos primitivos: Normales

Edad cronológica: 4 meses de edad.

Edad corregida: 1 meses 1 semana

Grupo 1 (desde los 2 meses hasta 1 día antes de cumplir los 3 meses).

Factores de riesgo biológico: (Enfermedades sistémicas de la madre, Gestación menor a 34 SDG, peso menor a $1,5 \mathrm{~kg}$, retardo en la respiración, hospitalización en UCIN).

Exploración neurológica: Alteración en la movilidad de su cuerpo.

Señales de alerta: Hipotonía, succión débil pero funcional, desarrollo general inadecuado.

Áreas del desarrollo: Motor grueso, motor fino, lenguaje (ROJO), social; VERDE.

Edad de desarrollo por desempeño: 0 - 1 mes.

Puntuación máxima: 1 Pasivo (en todos los criterios aplicables).

Puntuación media: 1 Pasivo (en todos los criterios aplicables)

Ítems no aplicables: Expresa sus logros, resuelve problemas, busca desafíos, modifica su ambiente, usa su imaginación).

Espacio de observación: Natural, interior, tranquilo. 


\section{Discusión}

El aumento de los bebés prematuros extremos ha desencadenado la necesidad de un seguimiento continuo e inmediato, mismo que sea pertinente y permita identificar las necesidades con base en el neurodesarrollo, para su intervención. En TO la perspectiva holística permite ampliar las modalidades de intervención, siendo punto de partida y de seguimiento elemental el proceso de evaluación.

Tabla 3 Resultados de intervención de TO en dos casos de prematuros extremos

\begin{tabular}{|c|c|c|c|}
\hline & Criterios & C1 & $\mathrm{C2}$ \\
\hline \multirow{6}{*}{$\begin{array}{l}\text { Evaluación } \\
\text { Neurológica para } \\
\text { niños menores } \\
\text { de cinco años } \\
\text { en el primer y } \\
\text { segundo nivel de } \\
\text { atención. }\end{array}$} & \multirow{10}{*}{$\begin{array}{l}{ }^{*} \text { Criterios de } \\
\text { normalidad y } \\
\text { anormalidad. }\end{array}$} & \multirow{3}{*}{$\begin{array}{l}\text { Estado de alerta: Normal } \\
\text { Postura de Reposo: Normal } \\
\text { Simetría de la expresión facial: } \\
\text { Normal }\end{array}$} & Estado de alerta: Normal \\
\hline & & & Postura de Reposo: Normal \\
\hline & & & $\begin{array}{l}\text { Simetría de la expresión facial: } \\
\text { Normal }\end{array}$ \\
\hline & & \multirow{2}{*}{$\begin{array}{l}\text { Seguimiento ocular y movimientos } \\
\text { oculares: Anormal (Estrabismo } \\
\text { corrección quirúrgica). }\end{array}$} & $\begin{array}{l}\text { Seguimiento ocular y movimientos } \\
\text { oculares: Normal }\end{array}$ \\
\hline & & & Reflejo Cocleopalpebral: Normal \\
\hline & & Reflejo Cocleopalpebral: Normal & Tono pasivo: Normal \\
\hline (11) & & \multirow{2}{*}{$\begin{array}{l}\text { Tono pasivo: Anormal, hipertonía } \\
\text { Ashworth } 1\end{array}$} & Tono activo: Anormal (sedente) \\
\hline \multirow{3}{*}{ Julio 2020} & & & Reflejos primitivos: Normales \\
\hline & & Tono activo: Anormal (sedente) & \\
\hline & & Reflejos primitivos: Normales & \\
\hline \multirow{3}{*}{$\begin{array}{l}\text { Evaluación } \\
\text { del Desarrollo } \\
\text { Infantil (EDI) }{ }^{(12)}\end{array}$} & \multirow{5}{*}{$\begin{array}{l}\text { Criterios de } \\
\text { clasificación del } \\
\text { Grupo C (desde los } \\
5 \text { meses hasta } 60 \\
\text { meses) }\end{array}$} & \multirow{3}{*}{$\begin{array}{l}\text { Edad cronológica: } 16 \text { meses de edad. } \\
\text { Edad corregida: } 14 \text { meses } \\
\text { Áreas del desarrollo: Motor grueso, } \\
\text { motor fino, lenguaje, personal social. } \\
\text { ROJO para edad cronológica y/o } \\
\text { corregida. }\end{array}$} & $\begin{array}{l}\text { Edad cronológica: } 8 \text { meses de } \\
\text { edad. }\end{array}$ \\
\hline & & & Edad corregida: 5 meses 1 semana \\
\hline & & & $\begin{array}{l}\text { Grupo } 5 \text { (desde los } 5 \text { meses hasta } \\
1 \text { día antes de cumplir los } 7 \text { meses). }\end{array}$ \\
\hline \multirow{2}{*}{ Julio 2020} & & \multirow[t]{2}{*}{$\begin{array}{l}\text { Edad de desarrollo por desempeño: } \\
7-9 \text { meses }\end{array}$} & $\begin{array}{l}\text { Áreas del desarrollo: Motor grueso, } \\
\text { motor fino (VERDE), lenguaje - } \\
\text { social (AMARILLO). }\end{array}$ \\
\hline & & & $\begin{array}{lccc}\text { Edad de desarrollo por } & \text { por } \\
\text { desempeño: } 5 & -6 \text { meses }\end{array}$ \\
\hline \multirow{5}{*}{$\begin{array}{l}\text { Cuestionario } \\
\text { Volicional } \\
\text { Pediátrico (CVP) } \\
\text { (13) }\end{array}$} & \multirow{5}{*}{$\begin{array}{l}14 \text { ítems } \\
\text { - Pasivo (1) } \\
\text { - Dudoso (2) } \\
\text { - Involucrado (3) } \\
\text { - Espontáneo (4) }\end{array}$} & $\begin{array}{l}\text { Puntuación máxima: } 4 \text { Espontáneo } \\
\text { (Muestra preferencias) }\end{array}$ & \multirow{2}{*}{$\begin{array}{l}\text { Puntuación máxima: } 4 \text { Espontáneo } \\
\text { (Muestra curiosidad, inicia acciones, } \\
\text { se orienta hacia un objetivo). }\end{array}$} \\
\hline & & \multirow{5}{*}{$\begin{array}{l}\text { Puntuación media: } 3 \text { Involucrado } \\
\text { (Muestra curiosidad, inicia acciones, } \\
\text { se orienta hacia un objetivo, intenta } \\
\text { cosas nuevas, permanece involucrado, } \\
\text { expresa placer de sus logros, trata de } \\
\text { producir efectos, práctica habilidades, } \\
\text { busca desafíos, modifica su ambiente) }\end{array}$} & \\
\hline & & & Puntuación media: 3 Involucrado \\
\hline & & & nuevas, permanece involucrado, \\
\hline & & & $\begin{array}{l}\text { trata de resolver problemas, práctica } \\
\text { habilidades, busca desafíos, }\end{array}$ \\
\hline \multirow[t]{3}{*}{ Julio 2020} & \multirow[t]{3}{*}{ - No aplicable (0) } & & modifica su ambiente) \\
\hline & & Ítems no aplicables: Usa la imaginación. & Ítems no aplicables: Usa su \\
\hline & & $\begin{array}{l}\text { Espacio de observación: Natural, } \\
\text { interior, tranquilo. }\end{array}$ & $\begin{array}{l}\text { Espacio de observación: Natural, } \\
\text { interior, tranquilo. }\end{array}$ \\
\hline
\end{tabular}


Los criterios de evaluación neurológica inicial están descritos en indicadores estructurales y funcionales, sin embargo, es importante considerar aspectos relacionados con actividades y participación, mismos que se pueden correlacionar adecuadamente con escalas del desarrollo infantil. En México, el proyecto de Norma Oficial 031 SSA2 2014 establece como obligatoria la evaluación neurológica y estimulación temprana ${ }^{14}$.

Promover el desempeño ocupacional en el niño posterior a una estancia prolongada en la UCIN, implica un reto en el abordaje, se debe buscar el proceso de interacción social, intencionalidad a través de la participación en experiencias con significado. El neurodesarrollo va más allá de facilitar la adquisición de hitos del neurodesarrollo

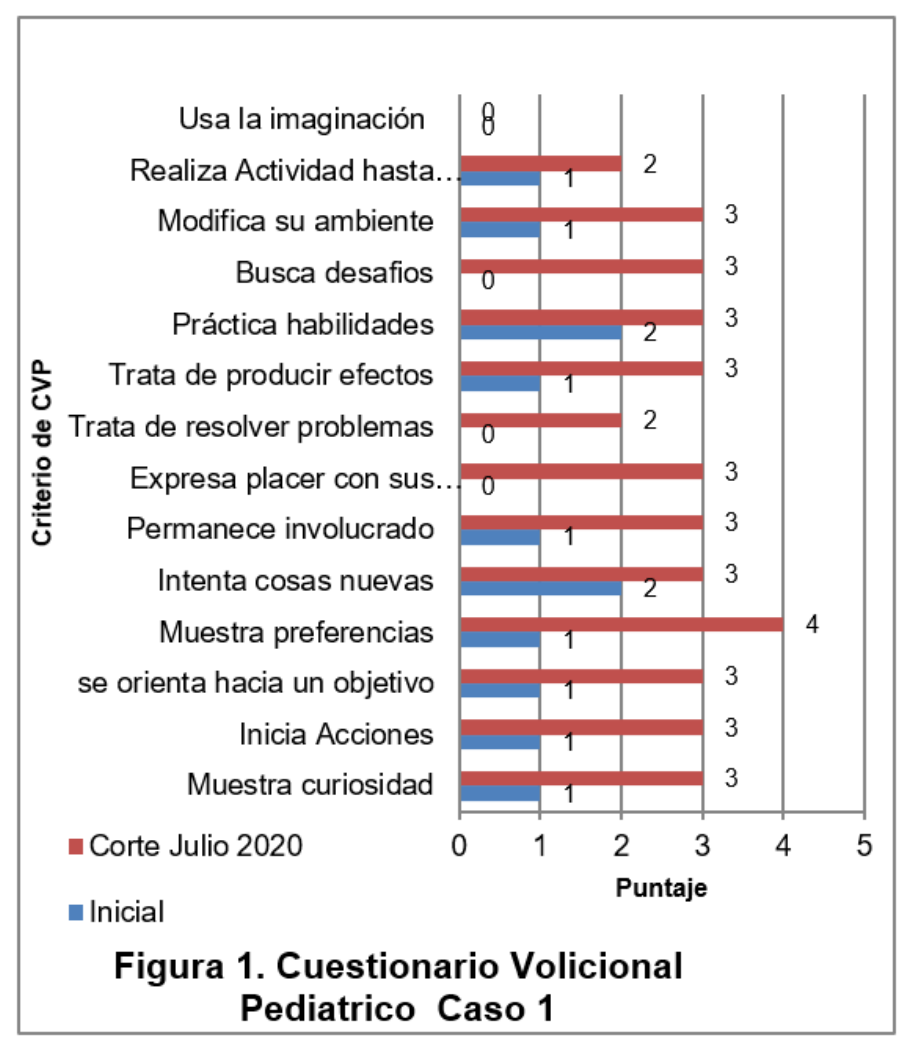

establecidos para estándares poblacionales y relacionados con el control motor. Se debe buscar secuencia de ellos, pero además estimular el desarrollo volicional, entendiéndolo como un ciclo constante de experimentar, interpretar, anticipar, y elegir la participación en actividades, el cual puede ser potenciado o limitado por el ambiente ${ }^{15}$.

La evolución del neurodesarrollo del niño no está relacionada con la frecuencia de intervención en terapia ocupacional, sino de factores de riesgo biológicos y de comorbilidad. En la actualidad, una de las metas para la consolidación de campos de conocimiento, atención y prospectiva es profundizar en la intervención que realiza el respectivo profesional en la UCIN.

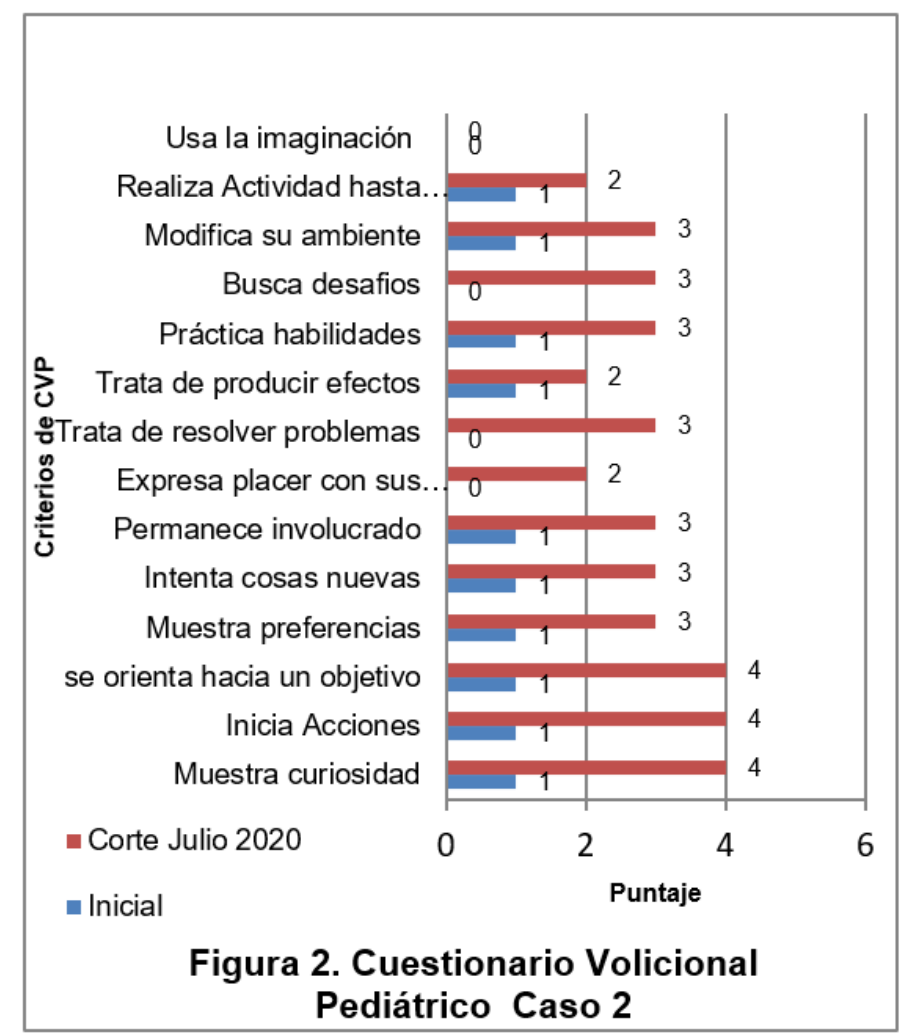




\section{Referencias Bibliográficas}

1. Avilés AC, Mandariaga SP, Fuentes LP, Venegas DC, Hurtado HR, Campos L. Intervención temprana en prematuros, una experiencia de trabajo. Rev Chil Ter Ocup. 2004;(4):11-18. doi: 10.5354/07195346.2010 .157

2. Organización Mundial de la Salud [Internet]. GInebra: OMS; 2018. Nacimientos prematuros; [aprox. 9 pantallas]. Disponible en: https://www.who.int/es/news-room/factsheets/detail/preterm-birth

3. Instituto Nacional de Pediatría (MX). Prematuridad y retos: prevención y manejo [Internet]. México: INP; 2018. Disponible en: https://pediatria.gob.mx/archivos/fasciculo_ prematurez.pdf

4. Instituto Mexicano de Seguridad Social. Cuidados del recién nacido prematuro sano hospitalizado [Internet]. México: IMSS; 2018. Disponible en: http://www.imss.gob. $\mathrm{mx/sites/all/statics/guiasclinicas/362GER.}$ pdf

5. Romero Sánchez J. Diferencias en el procesamiento sensorial entre niños/ as pretérmino y a término: el papel del terapeuta ocupacional. Rev Chil Ter Ocup. 2016;16(1):47-56. doi: 10.5354/07195346.2016 .41943

6. Mendoza LA, Arias M, Osorio MÁ. Factores asociados a estancia hospitalaria prolongada en neonatos. Rev Chil Pediatr. 2014;85(2):164-173. doi: 10.4067/S037041062014000200005
7. Polonio López B, Castellanos Ortega MC, Viana Moldes I. Terapia Ocupacional en la infancia: teoría y práctica. Madrid: Médica Panamericana; 2008.

8. World Federation of Occupational Therapists [Internet]. London: WFOT; 2012. About Occupational Therapy; [aprox. 3 pantallas]. Disponible en: https://www.wfot.org/ about/about-occupational-therapy

9. Boyt Schell A, Gillen G, Scaffa ME. Willard \& Spackman Terapia Ocupacional. Buenos Aires: Médica Panamericana; 2016.

10. Roley SS, DeLany JV, Barrows CJ, Brownrigg S, Honaker D, Sava DI, et al. Occupational therapy practice framework: domain \& practice, 2nd edition. Am J Occup Ther. 2008;62(6):625-83. doi: 10.5014/ ajot.62.6.625.

11. Comisión Nacional de Protección Social en Salud (MX). Manual de Exploración Neurológica para Niños Menores de Cinco Años en el Primer y Segundo Nivel de Atención México [Internet]. México: Secretaría de Salud; 2013. Disponible en: http://himfg. com.mx/descargas/documentos/EDI/ManualdeExploracionNeurologicaparaNinosMenoresde5enelPrimerySegundoNiveldeAtencion.pdf

12. Comisión Nacional de Protección Social en Salud (MX). Manual para la Aplicación de la Prueba Evaluación del Desarrollo Infantil "EDl". México: Secretaría de Salud; 2013. Disponible en: http://himfg.com.mx/ descargas/documentos/EDI/ManualparalaPruebadeEvaluaciondeIDesarrolloInfan- 


\section{til-EDI.pdf}

13. Basu S, Kafkes A, Geist R, Kielhofner G. Manual del Usuario del Cuestionario Volicional Pediátrico (PVQ) [Internet]. Chicago: University of Illinois; 1998. Disponible en: https://www.docdroid.net/7W47eCC/cuestionario-volicional-pediatrico-pdf

14. Proyecto de Norma Oficial Mexicana PROY - NOM -031-SSA2-2014. Para la atención a la salud de la infancia. Diario Oficial de la Federación (MX), (25 noviembre de 2015). Disponible en: https://www.dof.gob. $\mathrm{mx} /$ nota_detalle.php?codigo $=5417151 \& \mathrm{fe}-$ cha $=25 / 11 / 2015$

15. De las Heras G. Modelo de Ocupación Humana. Madrid: Síntesis; 2015. 